\title{
Observational Report
}

\section{Percutaneous Cement Vertebroplasty in the Treatment of Symptomatic Vertebral Hemangiomas}

Jie Hao, MD, PhD and ZhenMing Hu, MD, PhD

From: The First Affiliated Hospital, Chongqing Medical University, Chongqing, China.

Dr. Hao is Associate Professor of the Department of Orthopaedic Surgery, The First Affiliated Hospital, Chongqing Medical University, Chongqing,

China.

Dr. Hu is Professor and CoDirector of the Department

of Orthopaedic Surgery at

The First Affiliated Hospital, Chongqing Medical University, Chongqing, China.

Address correspondence: ZhenMing Hu, MD, PhD Professor \& Co-Director Department of Orthopaedic Surgery

The First Affiliated Hospital Chongqing Medical University 1 Youyi Rd

Chongqing 400016, China E-mail: zhenminghu62@yahoo.com.cn

Disclaimer: There was no external funding in the preparation of this manuscript.

Conflict of interest: None.

Manuscript received: 07/26/2011

Revised manuscript received: 10/20/2011

Accepted for publication: 11/03/2011

Free full manuscript: www.painphysicianjournal.com
Background: Vertebral hemangiomas are benign tumors with a rich vasculature. Symptoms may vary from simple vertebral pain, sometimes resistant to conservative medical treatment, to progressive neurological deficit. Surgery or radiotherapy have been the treatment of choice for several years, but they were worsened by intraoperative and postoperative hemorrhagic complications related to the rich vascularization that characterize these kinds of lesions, often preceded by a preoperative embolization in the acute setting. Recently, a percutaneous, minimally invasive technique of vertebroplasty has been introduced into clinical practice as an alternative to traditional surgical and radiotherapy treatment of symptomatic vertebral hemangiomas with or without features of aggressiveness at imaging studies.

Objective: This study aimed to illustrate the validity of treatment with percutaneous vertebroplasty (PVP) in patients with symptomatic vertebral hemangiomas (VHs).

Study Design: PVP in 26 patients with symptomatic VHs and its clinical effects were evaluated in 3-24 months follow-up.

Setting: An inteventional pain management practice, a medical center, major metropolitan city, China.

Methods: Twenty-six consecutive patients were treated with PVP; a total of 28 vertebral bodies. All patients were followed-up for 3-24 months, average 8.6 months. The clinical effects were evaluated with the visual analog scale (VAS) and 36-item short-form (SF-36) at preoperative and postoperative and final follow-up, comparing imaging before and post-treatment.

Results: Twenty-six patients (28 vertebral bodies) were treated successfully with a satisfying resolution of painful symptoms within 24 to 72 hours. Cement distribution was always diffuse and homogeneous. We found paravertebral cement leakage in 3 cases without any onset of radicular symptoms related to epidural diffusion. Spinal canal and intervertebral foramen cement leakage wasn't noticed. No pulmonary embolism ever occurred and no clinical and symptomatic complications were observed. Hemangioma was confirmed by pathology examination. VAS scores decreased from $7.5 \pm 1.5$ preoperatively to $1.6 \pm 0.6$ postoperatively, with a final score of $0.7 \pm 0.5$. There was significant difference between postoperative and preoperative, and between final followup and preoperative $(P<0.05)$. At the postoperative and final follow-up, the SF-36 scores of patients was significantly higher than the preoperative in Role Physical, Bodily Pain, General Health, Vitality, Social Functioning, Role Emotional, and Mental Health $(P<0.05)$.

Limitations: An observational study with a relatively small sample size.

Conclusions: PVP is an effective technique to treat symptomatic vertebral hemangioma, which is a valuable, minimally invasive, and quick method that allows a complete and lasting resolution of painful vertebral symptoms.

Key words: Percutaneous vertebroplasty, symptomatic, vertebral, hemangiomas.

Pain Physician 2012; 15:43-49 
V ertebral hemangiomas (VHs) are benign tumors with a rich vasculature and are a commonly encountered disease of the spine; most of them are asymptomatic. The predominant symptom is back pain (1). Some VHs can ultimately provoke medullary or radicular neurologic complications, requiring surgical excision (2-5). Surgical therapy has been for many years the treatment of choice for symptomatic or aggressive vertebral hemangiomas, which have been burdened with many complications related to intraoperative or postoperative bleeding and large trauma; furthermore, the procedure was difficult (6). Recently, a percutaneous, minimally invasive technique of vertebroplasty (PVP) has been introduced into clinical practice as an alternative to the traditional surgical and radiotherapy treatment of symptomatic vertebral hemangiomas with or without features of aggressiveness at imaging studies (7-10). Unfortunately, the data from published literature are mostly limited to case reports and small series of patients. In this report, we reviewed data on 26 patients with painful VH treated by PVP. The aim of this work is to illustrate the efficacy and safety of percutaneous cement vertebroplasty in the treatment of patient with symptomatic VHs.

\section{Methods}

\section{Case Information}

From January 2007 to March 2011, 26 consecutive patients were treated with PVP, for a total of 28 vertebral bodies affected by VHs: 17 thoracic vertebrae and 11 lumbar, including 18 women and 10 men, aged 2370 years, mean age was 49 years. The case history was one week to 11 years. All patients affected by VHs included in our cohort were symptomatic and resistant to conservative medical medications. The main symptom was vertebral pain without neurological deficit due to a vertebral fracture or medullary compression which was overcome or related to the extension of the lesion to the vertebral body/arch. Physical examination showed percussion pain in the spinal processes. Patients had no significant spinal kyphosis. Before surgery, they all received $\mathrm{x}$-ray, computed tomography (CT), and magnetic resonance imaging (MRI) tests. Combining imaging studies and physical examination, the responsible vertebra was determined (Figs. 1,2). Patients with systemic or local infections and those with noncorrectable coagulation disorders were ruled out from the treatment.

\section{Technique}

Local anesthesia was used. Patients were placed in the prone position. The body was supported by pads underneath the chest and pelvis and the abdomen was suspended. The PVP equipment of Shandong GuanLong Company (China) was used $(10,11)$. The puncture site was selected at pedicle of either the thoracic or lumbar vertebrae, and involved either unilateral or bilateral entries. Under guidance from a C-arm x-ray scan, the pedicle of the vertebra would be targeted and a mark would be made on its corresponding skin surface. Disinfection and draping occurred before the x-ray-guided, percutaneous puncture at the targeted site. Based on the $x$-ray at the occipital lateral position, the angle and direction of the needle would be adjusted before it was gradually advanced to $3-4 \mathrm{~mm}$ in front of the posterior cortex of the vertebral column. Gradually the puncture needle was advanced into the area of the vertebral body lesion. Then the puncture needle was removed and replaced by a guiding needle. The puncture needle capsule was then removed and replaced with a working capsule, where a fine, small drill head would be inserted into the lesion area to drill a canal in the vertebral body. Afterward, the drill head was removed. A biopsy forceps was inserted into the lesion area through the bone tunnel to take a small amount of lesion tissue for pathological examination. Bone cement was prepared into the "paste" stage. The cement introduction was always performed under fluoroscopic guidance with a slow and careful injection of high viscosity material. The amount of cement injected was variable, depending on the lesion that had to be treated. Approximately 4 to $7 \mathrm{~mL}$ of bone cement was injected. It was enough to fill up the lesion but not the entire vertebral body. A unipedicular approach for hemangioma of the thoracolumbar spine was performed in 10 patients and a bipedicular approach was performed in 16 patients. After surgery, the patient was ordered to lie down for 24 hours, before gradually sitting up and walking slowly.

\section{Assessment indices}

Results were evaluated using the visual analog scale (VAS) and 36-item short-form (SF-36) methods at preoperatively and postoperatively and at final followup, comparing imaging at pre- and post-treatment. All patients were followed up between 3 to 24 months, average 8.6 months.

Clinical effects were evaluated with VAS and SF-36 preoperatively and postoperatively and at final followup, comparing imaging at pre- and post-treatment. 

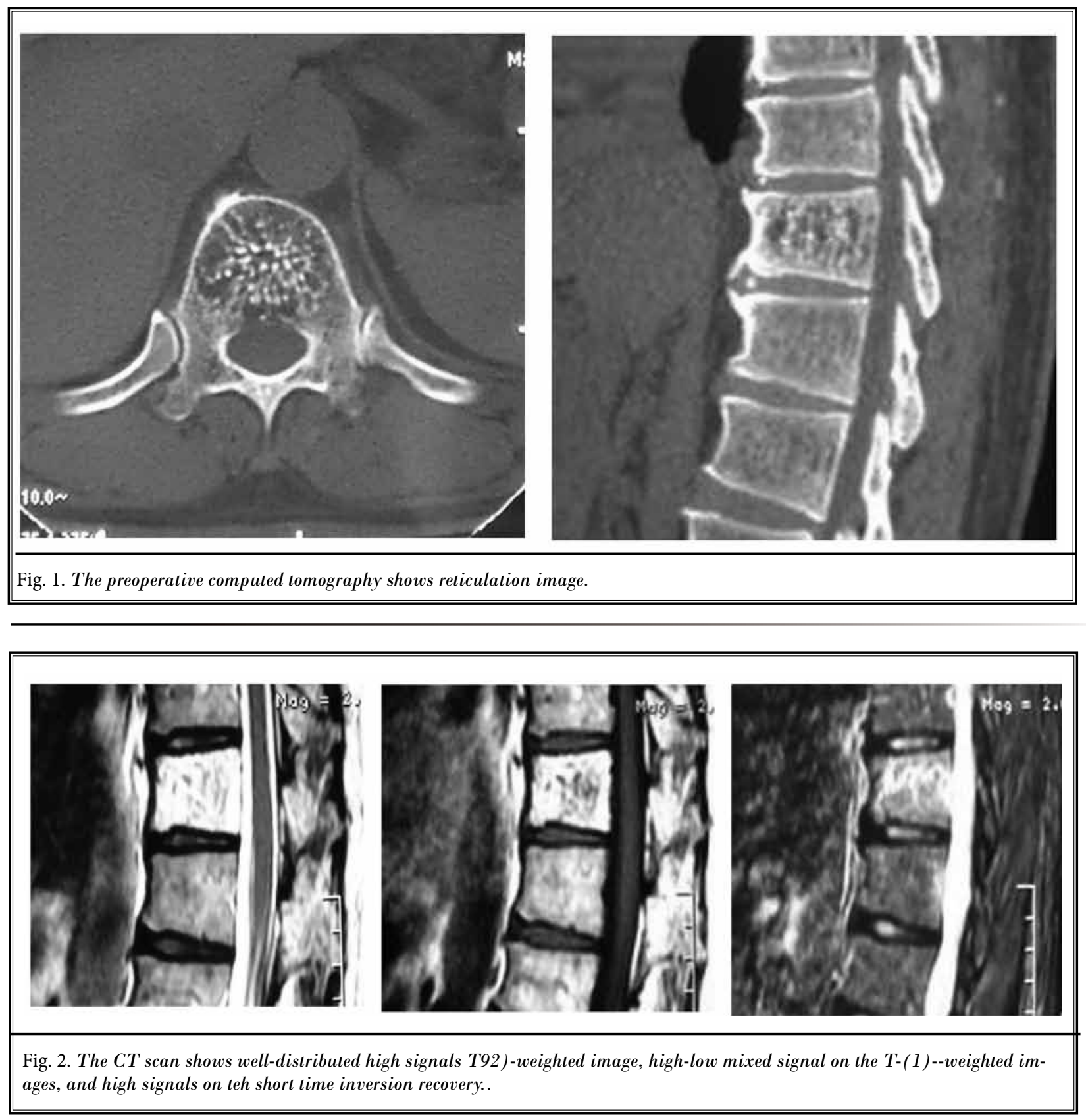

\section{Statistical analysis}

Data was presented in the form of $\bar{\chi} \pm s$ and processed by SPSS Version 11.0 (SPSS Inc., Chicago, IL). The pre-, post-operative, and final follow-up results were examined by using a paired $\mathrm{t}$ test. $P<0.05$ indicated statistical significance.

\section{Results}

There was a successful outcome in all patients with a satisfactory resolution of the painful symptoms within 24 to 72 hours. The dose of bone cement was about 4 to $7 \mathrm{~mL}$. The mean operative time was about 40 minutes. Intraoperative blood loss was one to $3 \mathrm{~mL}$. Cement distribution was always diffuse and homogeneous (Figs. 3,4) 

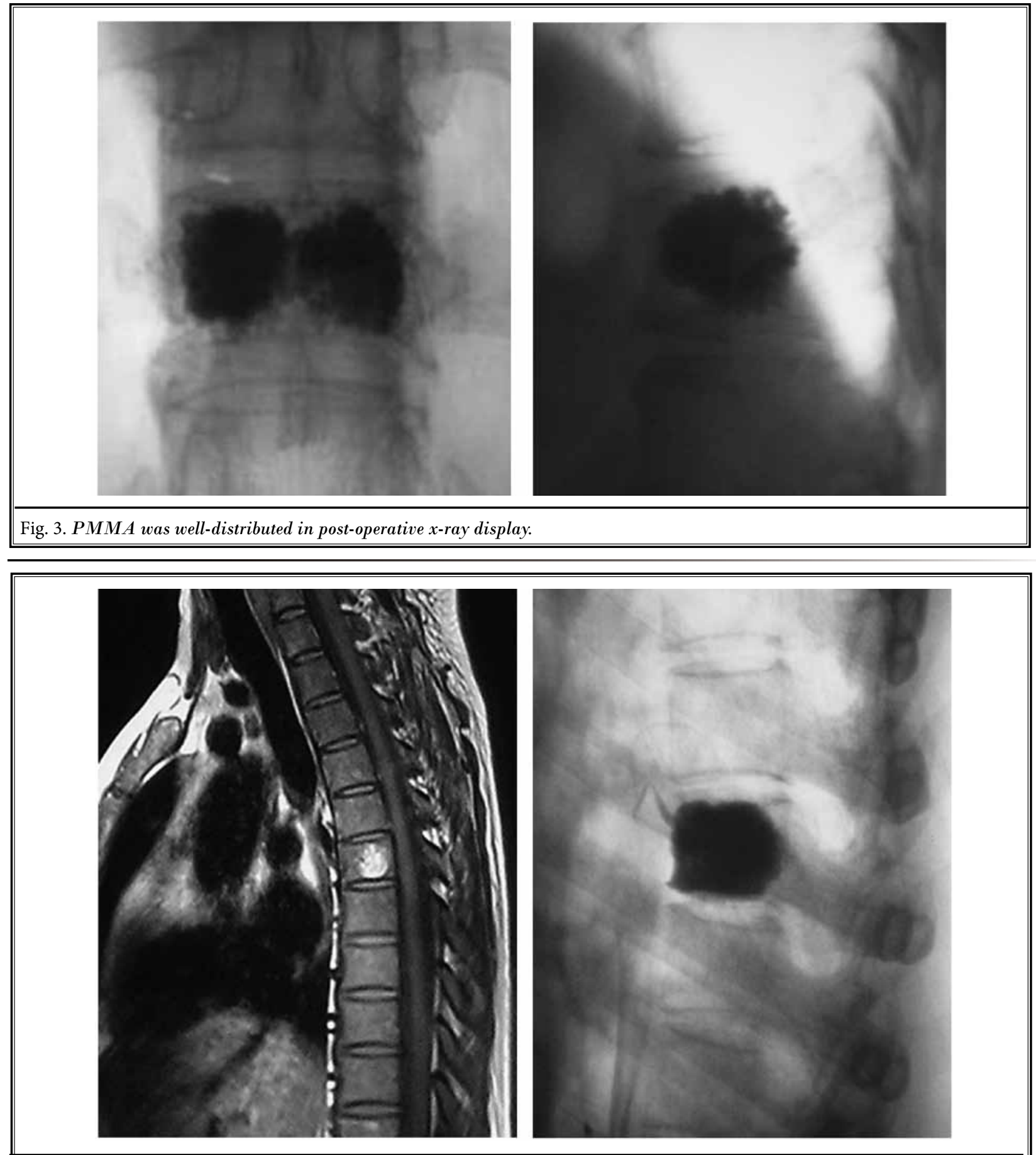

Fig. 4. The preoperative and postoperative computed tomography showed reticulation image.

We found paravertebral cement leakage in 2 cases without any onset of radicular symptoms related to epidural diffusion. Spinal canal and intervertebral foramen cement leakage was noticed in one 23-year-old woman; about $0.2 \mathrm{~mL}$ of cement leaked out into the left foraminal region and compressed the left nerve root. The only 
neurological complication was slight numbness in the leg. Immediately the spine posterior approach was used under local anesthesia.

A small incision was made to decompress and remove the cement particle; the patient's neurological symptoms were relieved. No pulmonary embolism ever occurred and no clinical or symptomatic complications were observed. Hemangioma was confirmed by pathology examination. Compared with the preoperative, VAS scores at postoperative and final follow-up were significantly reduced. VAS scores decreased from $7.4 \pm$ 1.6 preoperatively to $1.7 \pm 0.6$ postoperatively, with a final score of $0.7 \pm 0.4$. There was a significant difference between postoperative and preoperative, and between final follow-up and preoperative $(P<0.05)$ (Table 1). At the postoperative and final follow-up, the SF-36 scores of patients were significantly higher than the preoperative in Role Physical (RP), Bodily Pain (BP), General Health (GH), Vitality (VT), Social Functioning (SF), Role Emotional $(\mathrm{RE})$, and Mental Health $(\mathrm{MH})(P<0.05)$ (Table 2).

\section{Discussion}

VHs represent only 2-3\% of all spinal tumors with an incidence of $10-12 \%$ in the general population; only $0.9-1.2 \%$ of them are symptomatic (12). Asymptomatic patients with $\mathrm{VHs}$ are commonly detected during medical imaging examination for other diseases or during a health examination. Symptomatic patients complain of back pain. Physical examination shows percussion pain in spinal processes. In x-ray pictures, VHs appear with the typical "palisade" change. A CT cross-sectional image shows a low density area among sparse bone trabeculae in the focal zone, which looks like a honeycomb structure. MRI is highly sensitive to spinal hemangiomas. VHs with aggressive behavior present with low signal intensity on T1-weighted and high signal intensity on T2-weighted MRI, the typically signal characteristics demonstrates "benign" fatty stroma,it is bright on T1 and T2 weighted sequences and avidly enhances.

The extent of spinal cord compression and pathologic changes can be found by MRI $(1,13)$. The histological pattern is characterized by anomalous vascular proliferation with regular venous and capillary structures.

Currently, PVP has become an essential technique for the treatment of painful vertebral syndromes $(14,15)$. PVP is not commonly indicated for VHs due to the low incidence of this disease among the population. The correct and proper patient selection is related to a valid outcome for the PVP procedure. According to clinical manifestations, vertebral hemangiomas can be divided into 4 categories (8):

- Asymptomatic patient with no sign of aggressiveness

- Symptomatic patient with no sign of aggressiveness;

- Asymptomatic patient with signs of aggressiveness

- ASymptomatic patient with signs of aggressiveness.

There is no indication for PVP in the patients of the first group. The patients of the third group have to be carefully evaluated by radiological examination in or-

Table 1. VAS score of patients of vertebral hemangioma at pre-, post-operative and the final fellow-up $(\bar{X} \pm s)$

\begin{tabular}{|c|c|c|c|}
\hline & Preoperative & Postoperative & Final follow-up \\
\hline VAS & $7.4 \pm 1.6$ & $1.7 \pm 0.6$ & $0.7 \pm 0.4$ \\
\hline
\end{tabular}

Table 2. SF-36 score of patients of vertebral hemangioma at pre-, post-operative and the final fellow-up $(\overline{\mathrm{X}} \pm s)$

\begin{tabular}{|l|c|c|c|}
\hline & Preoperative & Postoperative & Final follow-up \\
\hline Physical Functioning ( PF) & $18.44 \pm 4.55$ & $27.35 \pm 3.48$ & $27.56 \pm 2.90$ \\
\hline Role Physical (RP) & $5.43 \pm 1.30$ & $7.17 \pm 1.47$ & $7.29 \pm 1.45$ \\
\hline Bodily Pain (BP) & $5.51 \pm 1.59$ & $9.27 \pm 2.34$ & $11.75 \pm 2.54$ \\
\hline General Health (GH) & $11.97 \pm 2.26$ & $17.31 \pm 2.11$ & $21.86 \pm 2.34$ \\
\hline Vitality (VT) & $14.79 \pm 2.66$ & $18.77 \pm 2.51$ & $20.02 \pm 2.92$ \\
\hline Social Functioning (SF) & $5.41 \pm 1.34$ & $6.17 \pm 1.02$ & $7.22 \pm 119$ \\
\hline Role Emotional (RE) & $4.35 \pm 0.81$ & $5.60 \pm 1.17$ & $5.71 \pm 0.92$ \\
\hline Mental Health) (MH) & $20.63 \pm 4.37$ & $23.47 \pm 2.12$ & $25.47 \pm 3.23$ \\
\hline
\end{tabular}


der to check the evolution of the disease. The second group could have an indication for PVP treatment due to the presence of low back pain. It is necessary to evaluate the radiological features of the epidural tissue and the cortical integrity of the responsible vertebral body.

Based on published data, the success rate of the procedure is $80-100 \%$ with a complication rate of $1-10 \%$. Leakage is one of the most common complications with risk of spinal cord compression or pulmonary embolism (16). Most patients had no clinical symptoms. The common leakage sites were the spinal canal, blood vessels, intervertebral discs, paravertebral, etc, which may accompany corresponding clinical symptoms (1718). In our study, 2 patients appeared to have paravertebral cement leakage without any symptomatic complications. Leakage of bone cement was associated with vertebral cortex integrity, operating skills, and the viscosity and dose of bone cement (9). An incomplete bone cortex increased the risk of bone cement leakage; the PVP procedure should be completed under the monitoring of a C-arm X-ray machine. The injection of cement must be done slowly and carefully. Once cement leakage is detected, the operator should adjust the needle direction or stop the injection immediately. The dose of bone cement depends on the size of the lesion (19), but we suggest enough bone cement be injected to fill the VH cave.

Because of possible problems with posterior vertebral cortex integrity due to osteoporosis in the study's elderly patients, the cement was always injected slowly and carefully into them. Usually, bone cement leakage did not occur. In the study's younger patients, the same caution was not followed and as a result, bone cement leakage sometimes happened. For example, one patient, a 23-year-old woman with an L4 hemangioma, had cement leakage into the left foraminal region. The injection was immediately stopped, but not before about $0.2 \mathrm{~mL}$ of cement had leaked, compressing the left nerve root. As a result, the patient felt a slight numbness in her leg. Under local anesthesia, a small incision was made and the cement particle was removed, relieving the numbness.

The principle of this technique is to fill completely the vertebral lesion with polymethylmethacrylate
(PMMA) cement in order to determine an irreversible sclerosis of the hemangiomatous venous pool, thus obtaining an antalgic effect. Local anesthesia was applied in PVP, which caused a light effects to the patients. The operator should observe changes in the patient's body sensation and movement of lower limbs during puncture and injection.

The monomer of bone cement is toxic and can cause anaphylaxis and blood pressure fluctuation. Based on the patient's weight, we administered 10-20 mg dexamethasone intravenously 5 minutes before the bone cement injection (10). As a result, 26 patients had no allergic reaction, sudden drop in blood pressure, or other adverse reaction.

PMMA possess no bone conductibility or biological activity. It can't be absorbed in the body; the interface of bone cement and bone could separate later. The operative vertebral body has higher mechanical strength than adjacent and distant vertebral body (11). Clinical results stayed stable without any occurrence of vertebral fractures in vertebral bodies adjacent or distant to the one treated in the 26 patients based upon standard anteroposterior and laterolateral X-rays.

\section{Conclusions}

PVP represents a therapeutic option available for the treatment of symptomatic vertebral hemangiomas based upon radiological evidence which are resistant to common conservative treatments. Compared with traditional surgery, PVP is a valuable minimally invasive tool; it is efficacious and safe and provides immediate pain relief and quick recovery of mobility in carefully selected patients. The mechanical-thermal effects of the cement leads to the destruction of the hemangioma, even if the tumor is not completely filled with cement. There is a minimal risk of bleeding. PVP provides an antalgic effect and vertebral stabilization and reduces the risk of secondary vertebral collapse.

\section{Acknowledgments}

The authors would like to thank the editors of Pain Physician journal for their review and constructive criticism in improving the manuscript. 


\section{References}

1. Urrutia J, Postigo R, Larrondo R Martin AS. Clinical and imaging findings in patients with aggressive spinal hemangioma requiring surgical treatment. J Clin Neurosci 2011; 18:209-212.

2. Kato S, Kawahara N, Murakami H, Demura S,Koshioka $Y$, Okayama T, Fujita $T$, Tomitam K. Surgical management of aggressive vertebral hemangiomas causing spinal cord compression: Longterm clinical follow-up of five cases. J Orthop Sci 2010; 15:350-356.

3. Shinozaki M, Morita A, Kamijo K, Seichi A, Saito $N$, Kirino $T$. Symptomatic $T_{2}$ vertebral hemangioma in a pregnant woman treated by one stage combination surgery; posterior stabilization and anterior subtotal tumor resection. Case report. Neurol Med Chir 2010; 50:674-677.

4. Aich RK, Deb AR, Banerjee A, Karim R, Gupta P. Symptomatic vertebral hemangioma: Treatment with radiotherapy. J Cancer Res Ther 2010; 6:199-203.

5. Heyd R, Seegenschmiedt MH, Rades D, Winkler C, Eich HT, Bruns F, Gosheger G, Willich N, Micke O. Radiotherapy for symptomatic vertebral hemangiomas: Results of a multicenter study and literature review. Int J Radiat Oncol Biol Phys 2010; 77:217-225.

6. Rich JA , Donahue TC, Mick TJ . Symptomatic expansile vertebral hemangioma causing conus medullaris compres- sion. J Manipulative Physiol Ther 2005; 28:194-198.

7. Jones JO, Bruel BM, Vattam SR. Management of painful vertebral hemangiomas with kyphoplasty: A report of two cases and a literature review. Pain Physician 2009; 12:297-303.

8. Guarnieri G, Ambrosanio G, Vassallo P, G.Pezzullo M, Galasso R, Lavanga A, Izzo $R$, Muto M. Vertebroplasty as treatment of aggressive and symptomatic vertebral hemangiomas: Up to 4 years of followup. Neuroradiology 2009; 51:471-476.

9. Brunot $S$, Berge J, Barreau X, Ménégon $P$, Dousset $V$. Long term clinical follow up of vertebral hemangiomas treated by percutaneous vertebroplasty. J Radiol 2005; 86:41-47.

10. Wang LJ, Hu ZM, Hao J. Percutaneous vertebroplasty in the treatment of symptomatic vertebral haemangiomas. Chin J Spine and Spinal Cord 2011; 3:256-257.

11. Hao J, Hu ZM,Meng CY. Percutaneous vertebroplasty for severely osteoporotic vertebral compression fractures. Chin J Orthop 2009; 17:884-886.

12. Laredo JD, Reizine D, Bard M. Vertebral hemangiomas: Radiologic evaluation. Radiology 1986; 161:183-189.

13. Elliott JM, Gorman I. Coincidental findings of a vertebral hemangioma on magnetic resonance imaging. J Orthop Sports
Phys Ther 2009; 39:149-154.

14. J ang JS, Lee SH. Efficacy of percutaneous vertebroplasty combined with radiotherapy in osteolytic metastatic spinal tumors. J Neurosurg Spine 2005; 2:243248.

15. Muijs SP, Dijkstra PD, van Erkel AR. Percutaneous vertebroplasty for vertebral fractures caused by multiple myeloma, an aggressive vertebral haemangioma and in a traumatic burst fracture [in Dutch]. Ned Tijdschr Geneeskd 2008; 26:1686-1693.

16. Evangelopoulos DS, Kontovazenitis P, Kokkinis K, Glynos M, Korres DS, Sapkas $\mathrm{G}$. Cement leakage in a symptomatic vertebral hemangioma: $A$ case report and review of the literature. Cases J 2009; 6:7148-7150.

17. Chen JK, Lee HM, Shih JT. Combined extraforaminal and intradiscal cement leakage following percutaneous vertebroplasty. Spine (Phila Pa 1976) 2007; 32:358-362.

18. Sonmez E, Yilmaz C, Caner H. Development of lumbar disc herniation following percutaneous vertebroplasty. Spine (Phila Pa 1976) 2010; 35:93-95.

19. Molloy S, Mathis JM, Belkoff SM. The effect of vertebral body percentage fill on mechanical behavior during percutaneous vertebroplasty. Spine (Phila Pa 1976) 2003; 28:1549-1554 
